\title{
Perturbational Imaging of Molecules with the Scanning Tunneling Microscope
}

Haoxuan Ding ${ }^{1}$, Jianzhi Gao ${ }^{2 *}$, Haoyu Zhao ${ }^{1}$, Chunqiu Xia ${ }^{1}$, Megan Grose ${ }^{1}$, Fangsen $\mathrm{Li}^{3 *}$, Quanmin Guo ${ }^{1}$

${ }^{1}$ School of Physics and Astronomy, University of Birmingham, Birmingham, B15 2TT, United Kingdom.

${ }^{2}$ School of Physics and Information Technology, Shaanxi Normal University, Xi'An 710119, China.

${ }^{3}$ Vacuum Interconnected Nanotech Workstation (Nano-X), Suzhou Institute of Nano-Tech and Nano-Bionics (SINANO), Chinese Academy of Sciences (CAS), Suzhou 215123, China.

A few more STM images are presented here. Figure S1 shows that individual $\mathrm{Au}$ atoms along the Au-adatom-row can be resolved. Figure S2 shows that for a constant tunnel current, switching the polarity of the bias voltage does not affect the image. Figure S3 shows that image switching can be achieved by altering the magnitude of the bias voltage. The tip-surface distance can be changed by either changing the bias at constant current or changing the tunnel current at constant bias.

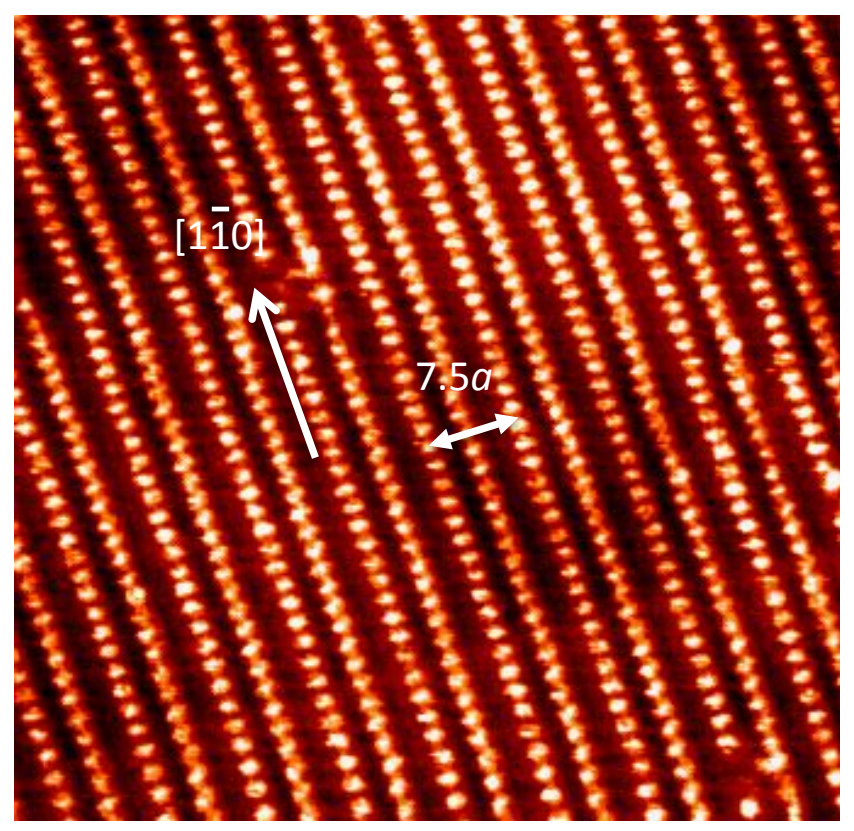

\footnotetext{
${ }^{*}$ Correspondence and requests for information should be addressed to Dr F-S Li, email: fsli2015@sinano.ac.cn. Prof J-Z Gao, Email: jianzhigao@snnu.edu.cn.
} 
Figure S1. Rows of $\mathrm{Au}$ adatoms observed with $-0.6 \mathrm{~V}$ sample bias and $0.05 \mathrm{nA}$ tunnel current. The $\mathrm{Au}$ adatoms are resolved as individual spots along the [112] direction. In the [1피 direction, the repeating period is $7.5 a$ where $a$ is the nearest neighbor distance of gold atoms. An adatom row is 3.5a from the neighboring row on its left and $4 a$ from the one on its right, or vice versa.
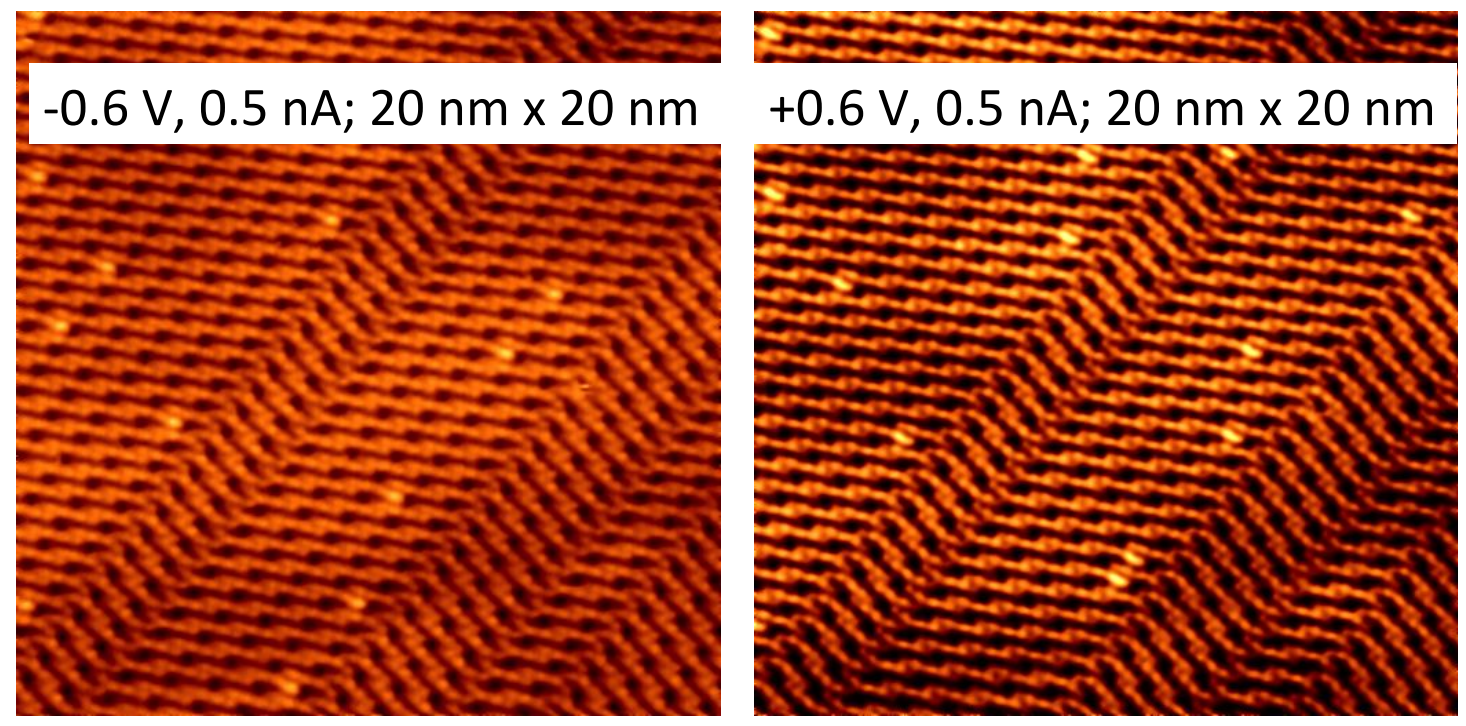

Figure S2. Switching the polarity of the bias voltage has no visible effect on the image.
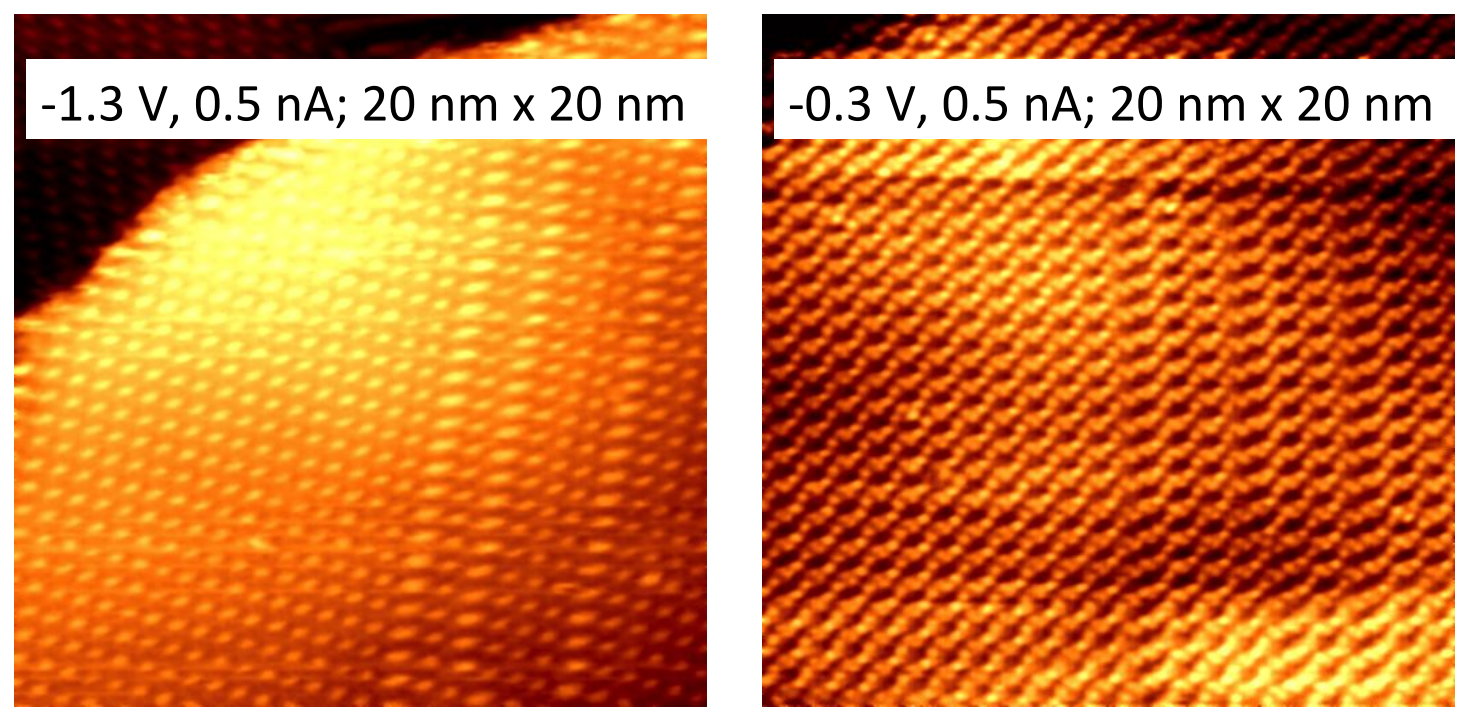

Figure S3. Keeping the tunnel current constant, the tip-surface distance can be changed by varying the magnitude of the bias voltage. This creates a similar effect as altering the tunnel current at constant bias voltage. The two images are collected from the same area of the sample. The image on the right is collected with the fast scan direction rotated anticlockwise by 19 degrees. 